\title{
Energy Efficiency Analysis of In-Building MIMO AF Communication
}

\author{
Fabien Héliot, Muhammad Ali Imran, and Rahim Tafazolli \\ Centre for Communication Systems Research, University of Surrey, \\ Guildford GU2 7HX, UK, Email: F.Heliot@Surrey.ac.uk
}

\begin{abstract}
Cooperative communication is an effective approach for increasing the spectral efficiency and/or the coverage of cellular networks as well as reducing the cost of network deployment. However, it remains to be seen how energy efficient it is. In this paper, we assess the energy efficiency of the conventional Amplifyand-forward (AF) scheme in an in-building relaying scenario. This scenario simplifies the mutual information formulation of the AF system and allows us to express its channel capacity with a simple and accurate closed-form approximation. In addition, a framework for the energy efficiency analysis of AF system is introduced, which includes a power consumption model and an energy efficiency metric, i.e. the bit-per-joule capacity. This framework along with our closed-form approximation are utilized for assessing both the channel and bit-per-joule capacities of the AF system in an in-building scenario. Our results indicate that transmitting with maximum power is not energy efficient and that AF system is more energy efficient than point-to-point communication at low transmit powers and signal-to-noise ratios.
\end{abstract}

Index Terms-Energy efficiency, cooperative communication, amplify-and-forward, MIMO systems.

\section{INTRODUCTION}

Energy efficiency has becoming a predominant criteria for designing reliable and low-power consumption communication networks in a context of ever growing energy demand and price. Up to now, the main design criteria was the spectral efficiency and as a result the energy consumption issues have received little attention, e.g. development of 3GPP systems. However, in the future mobile systems, e.g. Long Term Evolution-Advanced (LTE-A), the energy consumption will have to be taken into account in order to reduce the ever increasing running costs of the networks. One of the possible approaches to make networks more energy efficient is the use of cooperative communication [1]-[5]. Cooperative communication is a well-documented research area and it has proved to be an effective solution for increasing the spectral efficiency and/or the coverage of cellular networks as well as reducing the cost of network deployment [6]. The energy efficiency of single antenna relay system in low-power regime has been studied in [7]. However, the energy efficiency of cooperative multi-input multi-output (MIMO) AF has yet to be assessed and this is one of the aims of this paper.

Amplify-and-forward (AF) is a simple and practical approach for implementing cooperative MIMO communication. In $\mathrm{AF}$, the relay node ( $\mathrm{RN}$ ) simply amplifies the received signal from the source node (SN) and forwards it to the destination node (DN). In the original AF scheme [4], [8], the $\mathrm{RN}$ is used as a simple equal gain (EG) amplifier. In the general case, an intricate computable formula of the channel capacity for AF EG system has been derived in [9]. Whereas a simple asymptotic closed-form approximation of the channel capacity for AF EG system has been derived in [10] by assuming that the SN-DN link, i.e. direct link, is not active. In our study, we investigate the case where the RN-DN link is far more reliable than the other two links, which corresponds to an inbuilding relaying scenario, i.e a nonregenerative relay is used for relaying the signal of an outdoor base station to an indoor user [11]. We derive a close-form approximation of the channel capacity for this AF scenario by following a similar approach as in [10].

The rest of the paper is organized as follows. In Section II, we recall the generic cooperative MIMO AF system model, introduce the in-building scenario related assumptions and discuss their validity in terms of signal-to-noise ratio (SNR) of the RN-DN link. In Section III, we then provide an elegant and simple closed-form approximation of the channel capacity for the AF in-building scenario, which is utilized along with the power model of Section IV for assessing the energy efficiency of the AF EG system in Section V. In Section IV, we define a framework for the energy efficiency analysis of cooperative MIMO AF system by considering the power consumption model of [12] and the bit-per-joule capacity as an energy efficiency metric. Our results in Section $\mathrm{V}$ show that transmitting with maximum power is not energy efficient and also indicate the range of SNRs for which AF EG is more energy efficient than point-to-point (P2P) communication. Finally, conclusions are drawn in Section V.

\section{Cooperative MiMO AF System Model}

\section{A. General model}

We consider a cooperative MIMO AF system that is composed of three nodes, i.e. an SN with $n$ antennas, a nonregenerative RN with $q$ antennas and a DN with $r$ antennas, as depicted in Fig. 1. For the simplicity of the introduction, we assume a half-duplex relaying scenario with two phases of equal duration as in [5]. In the first phase, the SN broadcasts the signal $\mathbf{x}=\mathbf{R}$ s to the $\mathrm{DN}$ and $\mathrm{RN}$; in the second phase, only the RN transmits to the DN. Note that $\mathbf{R} \in \mathbb{C}^{n \times n}$ is the SN precoding matrix and $\mathrm{E}\left\{\mathbf{s s}^{\dagger}\right\}=\mathbf{I}_{n}$, where $\mathbf{I}_{n}$ is a $n \times n$ identity matrix and $\mathrm{E}\{$.$\} stands for the expectation. The signal$ $\mathbf{x}$ is received by the $\mathrm{DN}$ as $\mathbf{y}_{0}=\mathbf{H}_{0} \mathbf{x}+\mathbf{n}_{0}$ and by the RN as $\mathbf{y}_{1}=\mathbf{H}_{1} \mathbf{x}+\mathbf{n}_{1}$ at the end of the first phase. During the 


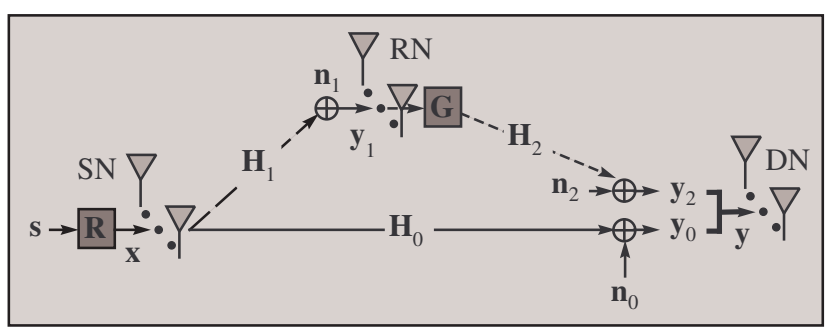

Fig. 1. MIMO AF communication system model.

second phase, the signal $\mathbf{y}_{1}$ is linearly precoded by using the precoding matrix $\mathbf{G} \in \mathbb{C}^{q \times q}$ at the $\mathrm{RN}$, is then transmitted towards the DN and is received as $\mathbf{y}_{2}=\mathbf{H}_{2} \mathbf{G} \mathbf{y}_{1}+\mathbf{n}_{2}$ by the DN. The matrices $\mathbf{H}_{0} \in \mathbb{C}^{r \times n}, \mathbf{H}_{1} \in \mathbb{C}^{q \times n}$ and $\mathbf{H}_{2} \in \mathbb{C}^{r \times q}$ represent the MIMO channels of the SN-DN, SN-RN and RNDN links, respectively. Each of these matrices is a random matrix having independent and identically distributed (i.i.d.) complex circular Gaussian entries with zero-mean and unit variance. In addition, $\mathbf{n}_{0} \in \mathbb{C}^{r \times 1}, \mathbf{n}_{1} \in \mathbb{C}^{q \times 1}$ and $\mathbf{n}_{2} \in \mathbb{C}^{r \times 1}$ are vectors of independent zero-mean complex Gaussian noise entries with a variance of $\sigma_{0}^{2}, \sigma_{1}^{2}$ and $\sigma_{2}^{2}$, respectively. The aggregate receive signal at the $\mathrm{DN}, \mathbf{y}$, can be expressed as

$$
\mathbf{y}=\left[\begin{array}{l}
\mathbf{y}_{0} \\
\mathbf{y}_{2}
\end{array}\right]=\left[\begin{array}{c}
\mathbf{H}_{0} \\
\mathbf{H}_{2} \mathbf{G H}_{1}
\end{array}\right] \mathbf{x}+\left[\begin{array}{ccc}
\mathbf{I}_{r} & \mathbf{0} & \mathbf{0} \\
\mathbf{0} & \mathbf{H}_{2} \mathbf{G} & \mathbf{I}_{r}
\end{array}\right]\left[\begin{array}{l}
\mathbf{n}_{0} \\
\mathbf{n}_{1} \\
\mathbf{n}_{2}
\end{array}\right] .
$$

The mutual information of the MIMO AF system is accordingly expressed as [13]

$$
I(\mathbf{y} ; \mathbf{s})=\frac{1}{2} \log _{2}\left|\mathbf{I}_{2 r}+\mathbf{H R R}^{\dagger} \mathbf{H}^{\dagger} \mathbf{R}_{\mathbf{n}}^{-1}\right|=\frac{1}{2} \log _{2}\left|\begin{array}{cc}
\mathbf{A} & \mathbf{D} \\
\mathbf{C} & \mathbf{B}
\end{array}\right|,
$$

where the factor $1 / 2$ accounts for the two-phase transmission, $(.)^{\dagger}$ denotes the conjugate transpose operator, $\mathbf{H} \in \mathbb{C}^{2 r \times n}$ characterizes the cooperative MIMO channel and $\mathbf{R}_{\mathbf{n}} \in$ $\mathbb{C}^{2 r \times 2 r}$ is the aggregate noise covariance matrix. Moreover, the matrices $\mathbf{A}, \mathbf{B}, \mathbf{C}$ and $\mathbf{D}$ are given by

$$
\begin{aligned}
& \mathbf{A}=\mathbf{I}_{r}+\mathbf{H}_{0} \mathbf{R} \mathbf{R}^{\dagger} \mathbf{H}_{0}^{\dagger} \mathbf{R}_{\mathbf{n}_{0}}^{-1} \\
& \mathbf{B}=\mathbf{I}_{r}+\mathbf{H}_{2} \mathbf{G}\left(\mathbf{R}_{\mathbf{y}_{1}}-\mathbf{R}_{\mathbf{n}_{1}}\right) \mathbf{G}^{\dagger} \mathbf{H}_{2}^{\dagger} \mathbf{R}_{\mathbf{n}_{2}}^{-1}, \\
& \mathbf{C}=\mathbf{H}_{2} \mathbf{G} \mathbf{H}_{1} \mathbf{R} \mathbf{R}^{\dagger} \mathbf{H}_{0}^{\dagger} \mathbf{R}_{\mathbf{n}_{0}}^{-1} \\
& \mathbf{D}=\mathbf{H}_{0} \mathbf{R} \mathbf{R}^{\dagger} \mathbf{H}_{1}^{\dagger} \mathbf{G}^{\dagger} \mathbf{H}_{2}^{\dagger} \mathbf{R}_{\mathbf{n}_{2}}^{-1}
\end{aligned}
$$

where $\mathbf{R}_{\mathbf{n}_{0}}=\sigma_{0}^{2} \mathbf{I}_{r}, \mathbf{R}_{\mathbf{n}_{2}}=\sigma_{2}^{2} \mathbf{I}_{r}+\mathbf{H}_{2} \mathbf{G R}_{\mathbf{n}_{1}} \mathbf{G}^{\dagger} \mathbf{H}_{2}^{\dagger}, \mathbf{R}_{\mathbf{n}_{1}}=$ $\sigma_{1}^{2} \mathbf{I}_{q}$ and $\mathbf{R}_{\mathbf{y}_{1}}=\mathrm{E}\left\{\mathbf{y}_{1} \mathbf{y}_{1}^{\dagger}\right\}=\mathbf{R}_{\mathbf{n}_{1}}+\mathbf{H}_{1} \mathbf{R} \mathbf{R}^{\dagger} \mathbf{H}_{1}^{\dagger}$ is the relay received signal covariance matrix. Furthermore, we can reexpress $I(\mathbf{y} ; \mathbf{s})$ as

$$
\begin{aligned}
I(\mathbf{y} ; \mathbf{s}) & =\frac{1}{2} \log _{2}|\mathbf{B}|+\frac{1}{2} \log _{2}\left|\mathbf{A}-\mathbf{D B}^{-1} \mathbf{C}\right| \\
& =\frac{1}{2} \log _{2}\left|\mathbf{I}_{n}+\mathbf{R}^{\dagger} \mathbf{R}_{\mathbf{y}} \mathbf{R}\right|
\end{aligned}
$$

by firstly applying the matrix determinant formula [14] to (2) and secondly by further expanding the first equation of (2) and using the matrix determinant inverse lemma [15]. In (4),

$$
\begin{aligned}
& \mathbf{R}_{\mathbf{y}}=\mathbf{H}_{0}^{\dagger} \mathbf{R}_{\mathbf{n}_{0}}^{-1} \mathbf{H}_{0}+\mathbf{H}_{1}^{\dagger} \mathbf{R}_{\mathbf{n}_{1}}^{-1}\left[\mathbf{I}_{q}-\mathbf{E}^{-1}\right] \mathbf{H}_{1} \\
& \mathbf{E}=\mathbf{I}_{q}+\left(\sigma_{1}^{2} / \sigma_{2}^{2}\right) \mathbf{G}^{\dagger} \mathbf{H}_{2}^{\dagger} \mathbf{H}_{2} \mathbf{G}
\end{aligned}
$$

\section{B. In-building relaying scenario}

In the following, we consider an equal power allocation at the $\mathrm{SN}$ and set $\mathbf{R}=\sqrt{P_{1} / n} \mathbf{I}_{n}$ such that $\operatorname{tr}\left(\mathbf{R} \mathbf{R}^{\dagger}\right)=P_{1}$, with $P_{1}$ being the total transmit power of the $\mathrm{SN}$ and $\operatorname{tr}($.) being the trace operator. In addition, we assume that the conventional AF EG scheme is employed at the RN such that $\mathbf{G}=\sqrt{P_{2} / \operatorname{tr}\left(\mathbf{R}_{\mathbf{y}_{1}}\right)} \mathbf{I}_{q}$ and $\operatorname{tr}\left(\mathbf{G R}_{\mathbf{y}_{1}} \mathbf{G}^{\dagger}\right)=P_{2}$, with $P_{2}$ being the total transmit power of the RN. Then, $\mathbf{E}$ in (5) can be re-expressed as $\mathbf{E}=\mathbf{I}_{q}+\alpha \mathbf{H}_{2}^{\dagger} \mathbf{H}_{2} / q$, where $\alpha=q\left(\sigma_{1}^{2} / \sigma_{2}^{2}\right)\left[P_{2} / \operatorname{tr}\left(\mathbf{R}_{\mathbf{y}_{1}}\right)\right]$, and clearly $\mathbf{E}^{-1} \simeq 0$ when $\alpha \gg 1$. Let us define $\bar{\alpha}$ as

$$
\bar{\alpha}=\mathrm{E}_{\mathbf{H}_{1}}\{\alpha\}=q\left(\frac{P_{2} \sigma_{1}^{2}}{\sigma_{2}^{2} q\left(P_{1}+\sigma_{1}^{2}\right)}\right)=\gamma_{2} /\left(\gamma_{1}+1\right),
$$

where $\gamma_{2}=P_{2} / \sigma_{2}^{2}$ and $\gamma_{1}=P_{1} / \sigma_{1}^{2}$ are the RN-DN and SNRN link SNRs, respectively. Inserting $\alpha=\bar{\alpha}$ in $\mathbf{E}$, it implies that $\mathbf{E}^{-1} \simeq 0$ on average when $\gamma_{2} \gg \gamma_{1}+1$, i.e. $\gamma_{2} \gg \gamma_{1}$. Consequently, $I(\mathbf{y} ; \mathbf{s})$ can be simplified as

$$
I(\mathbf{y} ; \mathbf{s}) \simeq \widetilde{I}(\mathbf{y} ; \mathbf{s})=\frac{1}{2} \log _{2}\left|\mathbf{I}_{n}+\lambda_{0} \mathbf{H}_{0}^{\dagger} \mathbf{H}_{0}+\lambda_{1} \mathbf{H}_{1}^{\dagger} \mathbf{H}_{1}\right|,
$$

when $\gamma_{2} \gg \gamma_{1}$, where $\lambda_{0}=P_{1} /\left(n \sigma_{0}^{2}\right)$ and $\lambda_{1}=P_{1} /\left(n \sigma_{1}^{2}\right)$. Thus, if the RN-DN link is far more reliable than the SNRN link, the cooperative mutual information simplifies into the expression in (7) for which a closed-form approximation of its expectation will be derived in the next section.

As stated above, equation (4) can be approximated as (7) as long as $\gamma_{2}(\mathrm{~dB})-\gamma_{1}(\mathrm{~dB}) \gg 0(\mathrm{~dB})$, i.e. $\gamma_{2}(\mathrm{~dB})=\gamma_{1}(\mathrm{~dB})+v$ $(\mathrm{dB}), v \gg 1$. In order to evaluate numerically the value of $v$ for which this approximation has a sufficient accuracy, we plot in Fig. $2 \Delta_{C}=C_{\mathrm{MC}}\left(\gamma_{2}=1 E^{10}\right)-C_{\mathrm{MC}}\left(\gamma_{2}\right)$ vs. $\gamma_{2}$, where $C_{\mathrm{MC}}\left(\gamma_{2}\right)$ is the expectation of $I(\mathbf{y} ; \mathbf{s})$ over $\mathbf{H}_{0}, \mathbf{H}_{1}$ and $\mathbf{H}_{2}$ that is obtained by using Monte-Carlo simulation. Moreover, $C_{\mathrm{MC}}\left(\gamma_{2}=10^{10}\right)$ corresponds to the expectation of $\widetilde{I}(\mathbf{y} ; \mathbf{s})$ over $\mathbf{H}_{0}$ and $\mathbf{H}_{1}$ that is also obtained by using Monte-Carlo simulation. Clearly, the results shows that $\Delta_{C}$ decreases as $\gamma_{2}$ increases. In addition, the various curves indicate that a difference of at most $0.1 \mathrm{bits} / \mathrm{s}$ can be achieved between the real capacity and its approximation as long as $v>20 \mathrm{~dB}$. In other words, our approximation in (7) becomes sufficiently accurate when $\gamma_{2}(\mathrm{~dB}) \geq \gamma_{1}(\mathrm{~dB})+20 \mathrm{~dB}$.

\section{Closed-Form APPROXIMATION OF THE MIMO AF CHANNEL CAPACITY}

\section{A. main result}

Here, we derive an accurate closed-form approximation of the MIMO AF capacity when $\gamma_{2} \gg \gamma_{1}$ by following a similar approach as in [10], which is based on random matrix theory and multiple saddle point integration technique. After intricate derivations, which are provided in [16], the MIMO AF channel capacity (in bits/s) can be approximated as

$$
\begin{aligned}
\widetilde{C} & =\frac{W}{2 \ln (2)}\left[n \ln \left(\frac{\gamma_{0}}{d_{0}}\right)+q\left(\ln \left(1+\sigma d_{0}\right)+\frac{1}{1+\sigma d_{0}},\right.\right. \\
& \left.-1)+r\left(\ln \left(1+d_{0}\right)+\frac{1}{1+d_{0}}-1\right)\right]
\end{aligned}
$$




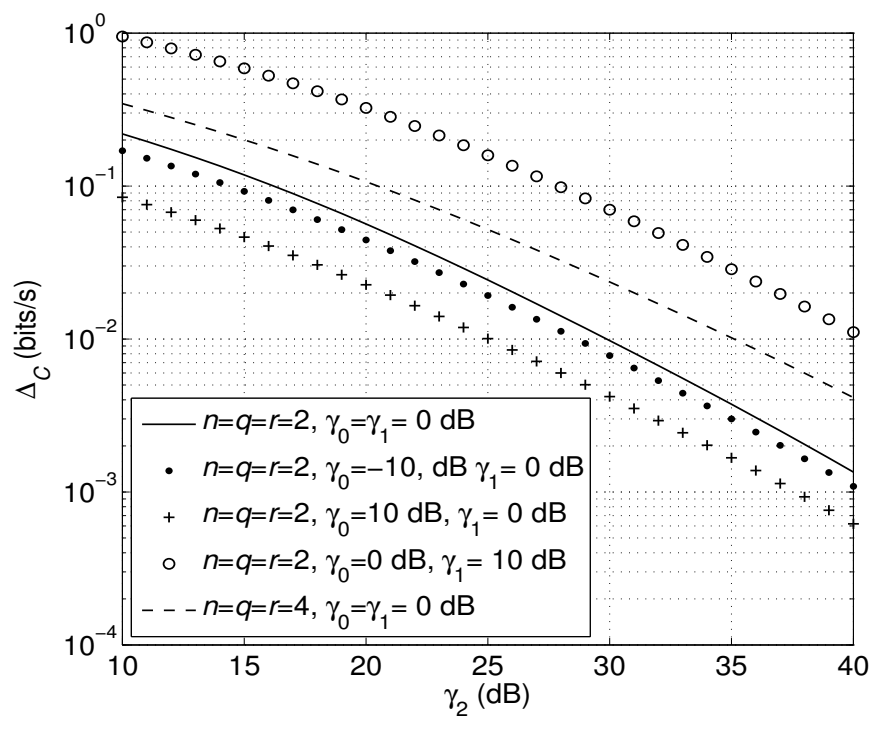

Fig. 2. Accuracy of our approximation in (7) according to $\gamma_{2}(\mathrm{~dB})$.

when $\gamma_{2} \gg \gamma_{1}$ and for large values of $n, q$ and $r$, where $\gamma_{0}=P_{1} / \sigma_{0}^{2}$ is the SN-DN link SNR, $\sigma=\gamma_{0} / \gamma_{1}=\sigma_{1}^{2} / \sigma_{0}^{2}$ is the SNR offset between the SN-DN and SN-RN links and $d_{0}$ is the unique nonnegative root of the polynomial $P(d)$ in (16) of [16]. The main purpose of (8) is the evaluation and comparison of the capacity of in-building MIMO AF systems in a faster way than time consuming Monte-Carlo simulations, and with a sufficient accuracy such that it can be used in network simulation and optimization. In addition, it can provide upper bounds on the achievable rate of generic cooperative MIMO AF systems.

The approximated channel capacity $\widetilde{C}$ is expressed as a function of the root $d_{0}$. According to equation (8) if $d_{0}$ is nonnegative then $\widetilde{C} \in \mathbb{R}$. It can be demonstrated that amongst the three roots of $P(d)$ only one of these roots is nonnegative and we set $d_{0}$ to be this root, such that

$$
\begin{aligned}
d_{0} & =\frac{1}{6}\left[-2 c_{1}+\frac{1-i \sqrt{3}}{\sqrt[3]{2}}\left(\sqrt[3]{A+i \sqrt{4 B^{3}-A^{2}}}\right)\right. \\
& \left.+\frac{1+i \sqrt{3}}{\sqrt[3]{2}}\left(\sqrt[3]{A-i \sqrt{4 B^{3}-A^{2}}}\right)\right]
\end{aligned},
$$

where $A=2 c_{1}^{3}-9 c_{1} c_{2}+27 c_{3}, B=c_{1}^{2}-3 c_{2}, c_{1}=\lambda_{0}(r+q-$ $n)+\sigma+1, c_{2}=\sigma\left[1+\lambda_{0}(r-n)+\lambda_{1}(q-n)\right]$ and $c_{3}=-n \lambda_{0} \sigma$.

\section{B. Accuracy analysis}

In order to assess numerically the accuracy of our approximation, we plot in Fig. $3 \Delta_{\epsilon} \triangleq 100\left|C_{\mathrm{MC}}-\widetilde{C}\right| / C_{\mathrm{MC}}$ vs. $\gamma(\mathrm{dB})$ for various $n, q$ and $r$ values, where $\Delta_{\epsilon}$ represents the difference between $C_{\mathrm{MC}}$ and $\widetilde{C}$ in percentage, and $C_{\mathrm{MC}}$ is the capacity value that is obtained by using Monte-Carlo simulation. We can clearly see in Fig. 3 that the accuracy of the approximation increases with the number of antennas at the SN, RN and DN. Moreover, the last curve indicates that the accuracy is also dependent on the SNR offset between the SN-RN and SN-DN links. However, a 99\% accuracy is at least

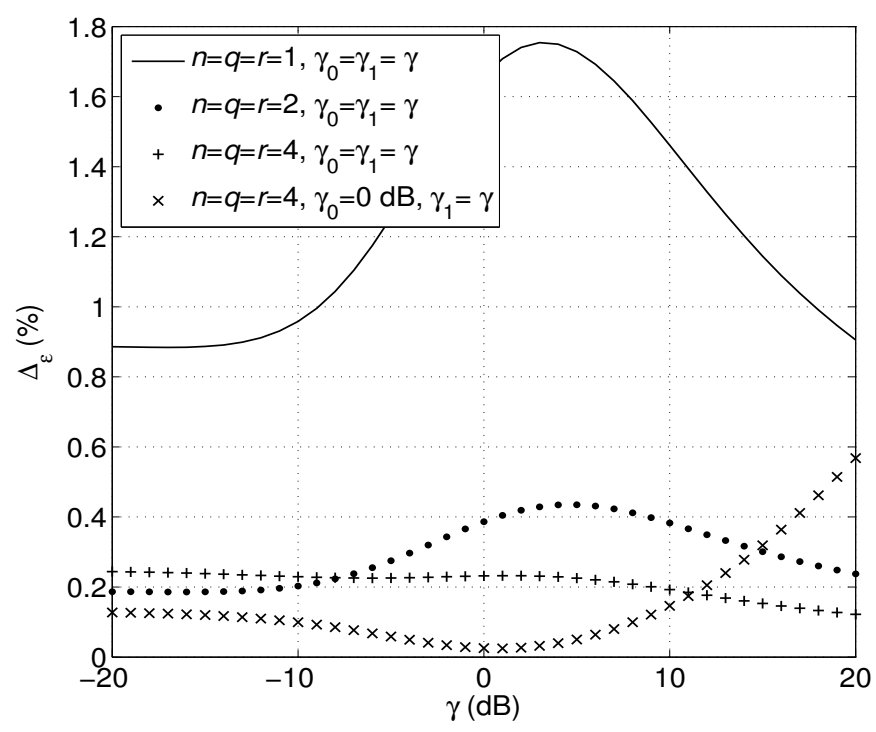

Fig. 3. Accuracy of $\widetilde{C}$ in (8) as a function of $\gamma(\mathrm{dB})$.

reached as long as $n=r=q \geq 2$ and regardless of the SNR offset. Thus, our approximation can be confidently used for swiftly assessing and comparing the capacity of MIMO AF.

\section{ENERGY EFFICIENCY ANALYSIS OF MIMO AF SYSTEM}

\section{A. Energy efficiency framework}

Traditionally, the efficiency of a communication system is measured in terms of spectral efficiency, which is related to the channel capacity in bits/s. This metric indicates how efficiently a limited frequency spectrum is utilized but does not provide any insight on how efficiently the energy is consumed. In order to evaluate this particular aspect of the communication system, we need a metric that takes into account the energy consumption. Such a metric, the bit-per-joule capacity (bits/J) has first been introduced in [17] and is simply defined as the ratio of the capacity to the rate of energy expenditure, i.e. to the total power of the system, such that

$$
C_{J}=C / P_{\mathrm{T}},
$$

where $P_{\mathrm{T}}$ is the total consumed power per transmission/reception. In our case we can also define

$$
C_{J} \approx \widetilde{C}_{J}=\widetilde{C} / P_{\mathrm{T}} .
$$

This metric indicates how efficiently energy is consumed for transmitting and receiving information. It has recently been used in [18] for analyzing the performance of energylimited wireless sensor and ad hoc networks. Here, it will be utilized for assessing the energy efficiency of MIMO AF communication.

In addition to this metric, a power consumption model is required to analysis the MIMO AF system in terms of energy efficiency. Since only the SN and RN transmit data in our scenario (see Section II), we consider that the total transmit consumed power $P_{\mathrm{T}}$ is such that

$$
P_{\mathrm{T}}=P_{\mathrm{SN}}+P_{\mathrm{RN}},
$$




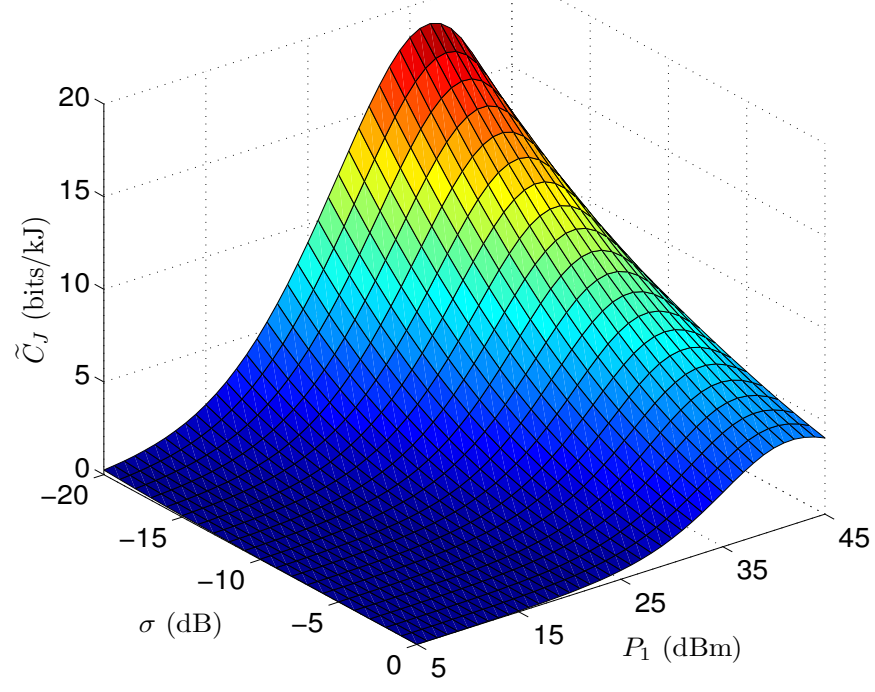

Fig. 4. Bit-per-joule capacity $\widetilde{C}_{J}$ as a function of the SNR offset $\sigma$ and the transmit power at the $\mathrm{SN} P_{1}$.

where $P_{\mathrm{SN}}$ and $P_{\mathrm{RN}}$ are the $\mathrm{SN}$ and RN consumed powers. Note that the received consumed power at the DN is considered negligible. We use the linear power consumption model for macro base station given in (1) of [12] for the SN,

$$
P_{\mathrm{SN}}=N_{\mathrm{Sector}} N_{\mathrm{PApSec}}\left(P_{1} / \mu_{\mathrm{PA} 1}+P_{\mathrm{SP}}\right)\left(1+C_{\mathrm{C}}\right)\left(1+C_{\mathrm{PSBB}}\right),
$$

where $N_{\text {Sector }}$ is the number of sector, $N_{\text {PApSec }}$ is the number of power amplifiers (PAs) per sector, $\mu_{\mathrm{PA} 1}$ is the PA efficiency, $P_{\mathrm{SP}}$ is the signal processing overhead, $C_{\mathrm{C}}$ is the cooling loss and $C_{\mathrm{PSBB}}$ is the battery backup and power supply loss. RN can be modelled as a micro, pico, or femto base station according to the scenario it is used in [11]. Here, we modelled the RN as a micro base station and use the linear power consumption model for micro base station given in (3) of [12] for the RN,

$$
P_{\mathrm{RN}}=\left(\left(P_{2} / \mu_{\mathrm{PA} 2}\right) C_{\mathrm{Tx}, \text { static }}+P_{\mathrm{SP}, \text { static }}\right)\left(1+C_{\mathrm{PS}}\right),
$$

where $\mu_{\mathrm{PA} 2}$ is the PA efficiency, $C_{\mathrm{Tx} \text {,static }}$ is the static transmit power coefficient, $P_{\mathrm{SP}}$ is the static signal processing overhead, $C_{\mathrm{PS}}$ is the power supply loss.

In our simulation, we have set the various parameters in (13) according to the values for UMTS1 in Table 3 of [12]. In addition, we have set $\mu_{\mathrm{PA} 2}=0.2, C_{\mathrm{Tx} \text {,static }}=1, P_{\mathrm{SP}}=15$ $\mathrm{W}$ and $C_{\mathrm{PS}}=0.11$ in (14), which are values also given in [12]. As for $P_{1}$ and $P_{2}$, we consider that $P_{1} \in\left[10^{-3}, \ldots, 40\right]$ $\mathrm{W}$, i.e $P_{1} \in[0, \ldots, 46] \mathrm{dBm}$, and $P_{2} \in\left[10^{-3}, \ldots, 5\right] \mathrm{W}$, i.e $P_{2} \in[0, \ldots, 37] \mathrm{dBm}$. Moreover, we set $W=1$ in (8)

\section{B. Numerical results for the in-building scenario}

By using our closed-form approximation along with the power consumption model described in Section IV-A, we can swiftly obtain theoretical energy efficiency limits for MIMO $\mathrm{AF}$ system with a given list of parameters $n, q, r, W, P_{1}, P_{2}, \gamma_{0}, \gamma_{1}, \gamma_{2}$, as shown in Fig. 4. Moreover, the comparison of theoretical limits of non-cooperative with

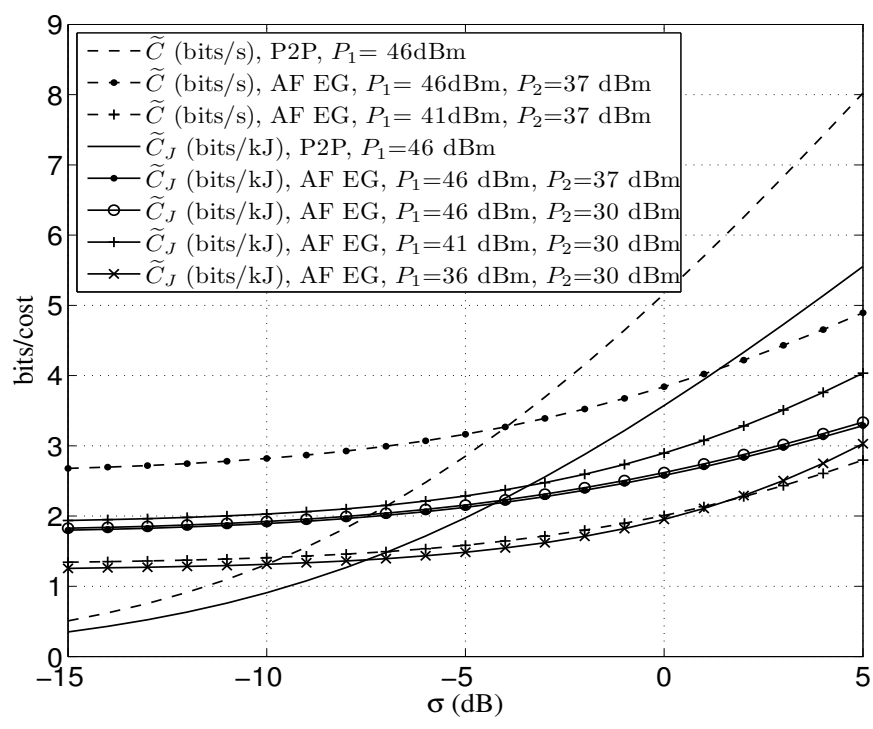

Fig. 5. Comparison of AF EG with P2P communication in terms of channel and bit-per-joule capacities as a function of $\sigma$.

cooperative systems can give us some insights on the energy efficiency of MIMO AF system. For instance, Figs. 5 and 6 compare the energy efficiency of a MIMO P2P system with a AF EG system in an in-building relaying scenario.

The results in Fig. 4 are plotted for $P_{1}$ varying from 5 to 45 $\mathrm{dBm}, \sigma$ varying from -20 to $0 \mathrm{~dB}, \sigma_{0}^{2}=40 \mathrm{dBm}, P_{2}=37$ $\mathrm{dBm}, \gamma_{2}=\gamma_{1}+20 \mathrm{~dB}$ and $n=q=r=4$. The results first clearly show that the maximum of the energy efficiency, $\widetilde{C}_{J, \max }$, is reached for $P_{1} \in[37, \ldots, 42] \mathrm{dBm}$, i.e $37 \mathrm{dBm}$ for $\sigma=-20 \mathrm{~dB}$ and $42 \mathrm{dBm}$ for $\sigma=0 \mathrm{~dB}$, and not for the maximum power $P_{1}=46 \mathrm{dBm}$, which provides the best spectral efficiency. The results also indicate that $\widetilde{C}_{J}$ increases as $\sigma$ decreases. Obviously, as $\sigma$ decreases, $\sigma_{1}^{2}$ decreases since $\sigma_{0}^{2}$ is fixed and, thus, $\widetilde{C}$ increases while $P_{\mathrm{T}}$ remains fixed, which makes $\widetilde{C}_{J}$ to increase as well. Moreover, $\widetilde{C}_{J \text {, max }}$ is reached for a lower $P_{1}$ values as $\sigma$ decreases. In the case that $\sigma=0 \mathrm{~dB}, \widetilde{C}_{J, \max }=4.57 \mathrm{bits} / \mathrm{kJ}$ is reached for $P_{1}=42 \mathrm{dBm}$ and $\gamma_{0}=\gamma_{1}=2 \mathrm{~dB}$. Whereas, the same energy efficiency can be obtained with 160 times less power, i.e., $P_{1}=20 \mathrm{dBm}$, when $\sigma=-20 \mathrm{~dB}, \gamma_{0}=-20 \mathrm{~dB}$ and $\gamma_{1}=0 \mathrm{~dB}$.

The results in Fig. 5 are plotted for $\sigma$ varying from -20 to $0 \mathrm{~dB}, \sigma_{1}^{2}=41 \mathrm{dBm}, \gamma_{2}=\gamma_{1}+20 \mathrm{~dB}, n=4, q=3$, $r=2$ and various values of $P_{1}$ and $P_{2}$. By comparing the first two curves, it becomes apparent that AF EG is more spectral efficient that P2P when $\gamma_{1}>\gamma_{0}+4 \mathrm{~dB}$ and the same remark can also be made in terms of energy efficiency by comparing the fourth with the fifth curve. Furthermore, by comparing the second, third, fifth and seventh curves, it reveals that the spectral efficiency of the AF EG system drops by around 40 to $50 \%$ when decreasing $P_{1}$ (down to $41 \mathrm{dBm}$ ) and, incidently $\gamma_{1}$; whereas its energy efficiency increases by 10 to $20 \%$. However, $\widetilde{C}_{J}$ will be reduced by further decreasing $P_{1}$ (down to $36 \mathrm{dBm}$ ). Finally, it can be noticed that $P_{2}$ has a limited impact on the variation of $\widetilde{C}_{J}$.

The results in Fig. 6 are plotted for $P_{1}$ varying from 20 to 46 


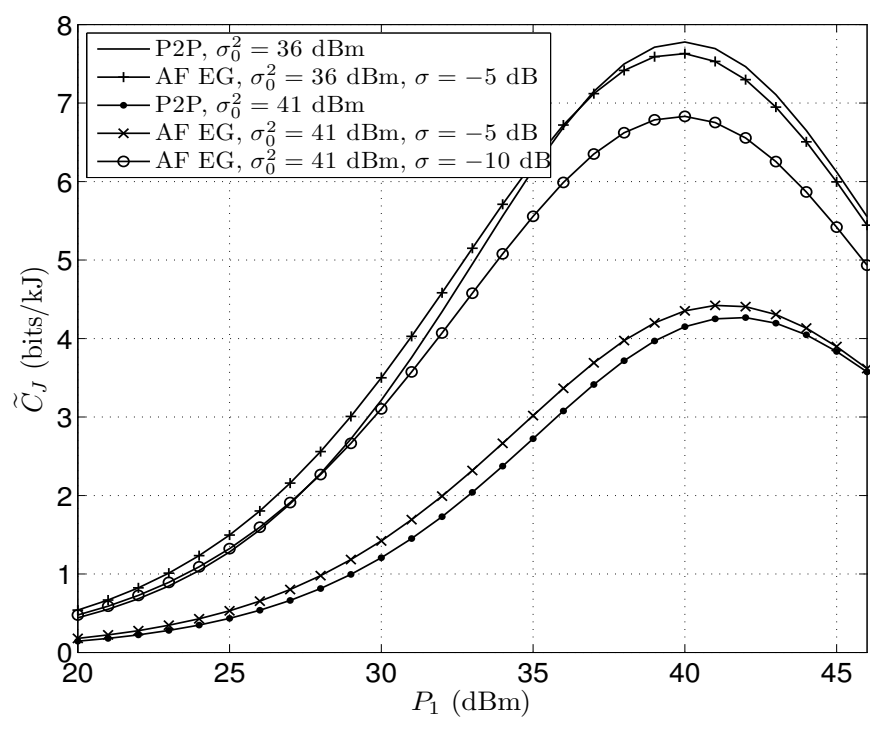

Fig. 6. Comparison of AF EG with P2P communication in terms of bit-perjoule capacity as a function of $P_{1}$.

$\mathrm{dBm}, \sigma_{1}^{2}=31 \mathrm{dBm}, P_{2}=37 \mathrm{dBm}, \gamma_{2}=\gamma_{1}+20 \mathrm{~dB}, n=4$, $q=3, r=2$ and various values of $\sigma_{0}^{2}$ and $\sigma$. The results indicate that AF EG is slightly more energy efficient than $\mathrm{P} 2 \mathrm{P}$ for low $P_{1}$ values whereas it is the contrary for high $P_{1}$ values when $\sigma=-5 \mathrm{~dB}$ and $\sigma_{0}^{2}=36 \mathrm{dBm}$. When $\sigma_{0}^{2}$ increases, i.e. $\gamma_{0}$ decreases, the energy efficiency gap between AF EG and P2P increases. Finally, it clearly becomes far more energy efficient to use AF EG when $\sigma$ increases, i.e. $\sigma_{0}^{2}$ increases.

Overall, the results in Figs 4, 5 and 6 indicate that even though transmitting with maximum power implies maximum spectral efficiency, however, it does not imply maximum energy efficiency. It also reveals that AF EG is more energy efficient than P2P communication as long as $\sigma \leq-5 \mathrm{~dB}$.

\section{CONCLUSiON}

In this paper, we have assessed the energy efficiency of MIMO AF communication for in-building relaying and shown that AF system is more energy efficient than $\mathrm{P} 2 \mathrm{P}$ communication at low transmit powers and SNRs and that transmitting with maximum power is not energy efficient.

Considering an in-building relaying scenario, the mutual information formulation of the MIMO AF system can be simplified and its channel capacity can be easily expressed via an accurate closed-form approximation. The accuracy of this approximation has been assessed for different numbers of antenna and SNR offset between the various links. Results have indicated that its accuracy is high, even for MIMO AF system with small numbers of transmit and receive antennas.

A framework for energy efficiency analysis of MIMO AF system has been introduced that includes a power consumption model and an energy efficiency metric. Our closed-form approximation of the AF MIMO capacity has been utilized for assessing both the channel and bit-per-joule capacities of in-building MIMO AF system. Results have indicated that AF
EG is more energy efficient than P2P communication as long as the direct link is $5 \mathrm{~dB}$ weaker than the SN-RN link.

In the future, we intend to refine the power model and generalize the analysis for any $\gamma_{2}$ values.

\section{ACKNOWLEDGMENT}

The research leading to these results has received funding from the European Commission's Seventh Framework Programme FP7/2007-2013 under grant agreement n ${ }^{\circ} 247733$ project EARTH.

\section{REFERENCES}

[1] A. Sendonaris, E. Erkip, and B. Aazhang, "User Cooperation Diversity Part I \& II- System Description / Implementation Aspects and Performance Analysis," IEEE Trans. Commun., vol. 51, no. 11, pp. 1927-1948, Nov. 2003.

[2] M. Janani, A. Hedayat, T. E. Hunter, and A. Nosratinia, "Coded Cooperation in Wireless Communications: Space-Time Transmission and Iterative Decoding," IEEE Trans. Signal Processing, vol. 52, no. 2, pp. 362-371, Feb. 2004.

[3] A. Nosratinia, T. E. Hunter, and A. Hedayat, "Cooperative Communication in Wireless Networks," IEEE Commun. Mag., vol. 42, no. 10, pp. 74-80, Oct. 2004.

[4] J. N. Laneman, D. N. C. Tse, and G. W. Wornell, "Cooperative Diversity in Wireless Networks: Efficient Protocols and Outage Behavior," IEEE Trans. Inform. Theory, vol. 50, no. 12, pp. 3062-3080, Dec. 2004.

[5] O. Muñoz-Medina, J. Vidal, and A. Agustín, "Linear Transceiver Design in Nonregenerative Relays with Channel State Information," IEEE Trans. Signal Processing, vol. 55, no. 6, pp. 2593-2604, June 2007.

[6] "Reconfigurable OFDMA-based Cooperative NetworKs Enabled by Agile SpecTrum Use (ROCKET)," ICT-215282 FP7 STREP project, Tech. Rep., 2008-2009. [Online]. Available: http://www.ict-rocket.eu

[7] Y. Yao, X. Cai, and G. B. Giannakis, "On Energy Efficiency and Optimum Resource Allocation of Relay Transmissions in the Low-Power Regime," IEEE Trans. Wireless Commun., vol. 4, no. 6, pp. 2917-2927, Nov. 2005.

[8] K. Azarian, H. El Gamal, and P. Schniter, "On the Achievable DiversityMultiplexing Tradeoff in Half-Duplex Coooperative Channels," IEEE Trans. Inform. Theory, vol. 51, no. 12, pp. 4152-4172, Dec. 2005.

[9] A. Firag, P. J. Smith, and M. R. McKay, "Capacity Analysis for MIMO Two-Hop Amplify-and-Forward Relaying Systems with the Source to Destination Link,' in Proc. IEEE ICC'09, Dresden, Germany, June 2009.

[10] J. Wagner, B. Rankov, and A. Wittneben, "Large n Analysis of Amplifyand-Forward MIMO Relay Channels With Correlated Rayleigh Fading," IEEE Trans. Inform. Theory, vol. 54, no. 12, pp. 5735-5746, Dec. 2008.

[11] A. Ambrosy and et al., "D2.2: Definition and Parameterization of Reference Systems and Scenarios," INFSO-ICT-247733 EARTH (Energy Aware Radio and NeTwork TecHnologies), Tech. Rep., June 2010. [Online]. Available: https://www.ict-earth.eu/publications/ deliverables/deliverables.html

[12] O. Arnold, F. Richter, G. Fettweis, and O. Blume, "Power Consumption Modeling of Different Base Station Types in Heterogeneous Cellular Networks," in Proc. ICT Future Network \& Mobile Summit, Florence, Italy, June 2010.

[13] T. M. Cover and J. A. Thomas, Elements of Information Theory. NewYork, USA: ed. Wiley, 1991.

[14] "Imperial College Website, Block Matrix Determinant," [Online]. Available: http://www.ee.ic.ac.uk/hp/staff/dmb/matrix/ proof003.html.

[15] "Wikipedia Website," [Online]. Available: http://en.wikipedia.org/wiki/Matrix_determinant_lemma.

[16] F. Héliot, M. A. Imran, and R. Tafazolli, "Energy Efficiency Analysis of In-Building MIMO AF Communication: Derivation Insights," Tech. Rep., Aug. 2010. [Online]. Available: http://membres.multimania.fr/ fheliot/pub/Technote5.pdf

[17] H. M. Kwon and T. G. Birdsall, "Channel Capacity in Bits per Joule," IEEE Jour. Oceanic Engineering, vol. OE-11, no. 1, pp. 97-99, Jan. 1986.

[18] V. Rodoplu and T. H. Meng, "Bits-per-Joule Capacity of Energy-Limited Wireless Networks," IEEE Trans. Wireless Commun., vol. 6, no. 3, pp. 857-865, Mar. 2007. 